ARTICLE

\title{
FIS1 encodes a GA2-oxidase that regulates fruit firmness in tomato
}

\author{
Ren $\mathrm{Li}^{1,2,7}$, Shuai Sun ${ }^{1,2,7}$, Haijing Wang ${ }^{1,2}$, Ketao Wang ${ }^{3}$, Hong Yu (1) 4, Zhen Zhou ${ }^{1,2}$, Peiyong Xin ${ }^{4}$, Jinfang Chu ${ }^{4}$,

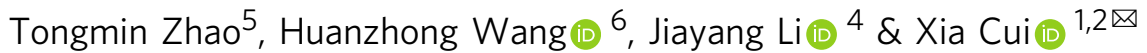

Fruit firmness is a target trait in tomato breeding because it facilitates transportation and storage. However, it is also a complex trait and uncovering the molecular genetic mechanisms controlling fruit firmness has proven challenging. Here, we report the map-based cloning and functional characterization of qFIRM SKIN 1 ( $q F I S 1$ ), a major quantitative trait locus that partially determines the difference in compression resistance between cultivated and wild tomato accessions. FIS1 encodes a GA2-oxidase, and its mutation leads to increased bioactive gibberellin content, enhanced cutin and wax biosynthesis, and increased fruit firmness and shelf life. Importantly, FIS1 has no unfavorable effect on fruit weight or taste, making it an ideal target for breeders. Our study demonstrates that FIS1 mediates gibberellin catabolism and regulates fruit firmness, and it offers a potential strategy for tomato breeders to produce firmer fruit.

\footnotetext{
${ }^{1}$ Key Laboratory of Biology and Genetic Improvement of Horticultural Crops of the Ministry of Agriculture, Institute of Vegetables and Flowers, Chinese Academy of Agricultural Sciences, Beijing 100081, China. ${ }^{2}$ Sino-Dutch Joint Laboratory of Horticultural Genomics, Institute of Vegetables and Flowers, Chinese Academy of Agricultural Sciences, Beijing 100081, China. ${ }^{3}$ State Key Laboratory of Subtropical Forest Cultivation, Zhejiang Agriculture and Forestry University, Hangzhou 311300, China. ${ }^{4}$ State Key Laboratory of Plant Genomics and National Center for Plant Gene Research (Beijing), Institute of Genetics and Developmental Biology, Chinese Academy of Sciences, Beijing 100101, China. ${ }^{5}$ Vegetable Research Institute, Jiangsu Academy of Agricultural Science, Nanjing 210014, China. ${ }^{6}$ Department of Plant Science and Landscape Architecture, University of Connecticut, Storrs, CT 06269, USA. ${ }^{7}$ These authors contributed equally: Ren Li, Shuai Sun. ${ }_{\text {email: cuixia@caas.cn }}$
} 
$\mathrm{T}$ omato (Solanum lycopersicum) is one of the most important vegetable crops worldwide and has a net production value of over $\$ 55$ billion ${ }^{1}$. Squashed and softened fruits are the most common defects and have tremendous associated costs in terms of transportation and storage ${ }^{2}$. The ripening inhibitor (rin) mutation has been introduced into hybrids to produce firm fruits with a long shelf life and improved transportability, but these fruits often have poor taste and are slow to turn color ${ }^{3,4}$. Therefore, enhanced fruit firmness that does not compromise other fruit qualities is a key target characteristic in modern tomato breeding.

Fruit firmness is a complex trait involving numerous physical properties, including cell-wall structure, cellular turgor, and cuticle characteristics $^{5-8}$. Disintegration and degradation of the cell wall are intimately involved in fruit softening. These events are accompanied by the increased expression of genes encoding cell-wall degrading enzymes, including polysaccharide hydrolases, transglycosylases, and other modification proteins, which influence the cell-wall components, resulting in a change in fruit firmness ${ }^{2}$. In addition, the thickness of the cuticle coordinated with expansion of the fruit surface is gradually increased to maintain fruit epidermal structural integrity during fruit growth ${ }^{9,10}$. These dynamic changes in cuticle architecture and composition were proven to be integral elements for fruit firmness alteration ${ }^{7,9}$. Fruit cuticles as composite structures are composed mainly of polymer cutin and cuticular wax ${ }^{11}$. Reducing the contents of these components results in a thinner cuticle, which leads to a decrease in fruit water retention, shelf life, and fruit firmness $9,10,12$. Therefore, the intricate mechanisms underlying fruit firmness need to be disentangled and characterized to effectively manipulate the trait in breeding.

In this regard, several quantitative trait loci (QTLs) associated with firmness have been identified ${ }^{13-15}$. To date, only one locus has been mapped to a $8.6 \mathrm{Mb}$ region on tomato chromosome 2 using introgression lines (ILs), and three pectin methylesterases were nominated as candidate genes ${ }^{15}$. However, precisely identifying the genes associated with fruit firmness remains a daunting task. In recent years, the roles of some genes in fruit firmness have been certified by transgenic tomatoes. Silencing the pectate lyase $(P L)$ gene in tomatoes enhances fruit firmness and prolongs shelf life ${ }^{5}$. Overexpression or silencing of the TAGL1 gene in tomato influences cuticle thickness, which is coordinated with the alteration of fruit firmness ${ }^{16-18}$. Although these studies provided usable genes for improving fruit firmness, the limited knowledge of the genes and mechanisms related to fruit firmness still impedes trait selection in breeding.

Gibberellins (GAs) are crucial for a wide range of developmental processes in higher plants. In tomato, GAs likely regulate tomato fruit growth. The application of exogenous $\mathrm{GA}_{3}$ to unpollinated ovaries induced fruit set ${ }^{19}$. Transgenic approaches have also proven that changes in GAs levels in tomatoes can induce fruit parthenocarpic development and affect fruit ripening $^{20-22}$. However, as an important hormone, the effect of GAs on other developmental processes of tomato fruit is still unknown. We identified a quantitative trait locus, qFIRM SKIN 1 ( $q$ FIS1), that determines fruit firmness between wild and cultivated tomatoes. FIS1 encodes a GA2-oxidase, and its mutation resulted in an increase in bioactive gibberellin contents in fruits that caused an increase in fruit firmness due to changes in cuticle composition and deposition in the tomato pericarp. Exogenous gibberellin treatment rescued the compression resistance of NILFIS1 ${ }^{\mathrm{CC}}$ fruits accompanied by an increase in cuticle thickness. The presence of the fis1 allele was selected during tomato domestication and contributed to the higher fruit firmness of modern cultivars. Knockout of FIS1 could increase fruit firmness in tomato. Therefore, the discovery of FIS1 provides new insight into the mechanism of gibberellin for fruit firmness, making this gene an ideal target for improving fruit firmness in many crops.

\section{Results}

qFIS1 is a major locus contributing to fruit firmness in tomato. As a complex trait, fruit firmness is very difficult to quantify, which inhibits the identification of loci. We used a texture analyzer to evaluate compression resistance (CR), which is defined as the pressure when a compressed fruit is broken, reflecting the fruit resistance to squeezing force (Fig. 1a) ${ }^{23}$. We compared the CR of two tomato accessions, Solanum lycopersicum var. cerasiforme LA1310 (CC) and S. lycopersicum Moneymaker (MM), and found that MM had significantly higher resistance to squeezing (Fig. 1b). We then systematically measured the CR of a stable recombinant inbred line (RIL) population, including more than 200 independent lines derived from these two parents ${ }^{24}$. The distribution of CR resembled an approximately normal distribution (Supplementary Fig. 1a), and two replications showed a high correlation (Supplementary Fig. 1b). An associated study using the whole-genome sequencing data of the RILs identified one significant signal, named qFIS1 (QTL of FIRM SKIN), on the short arm of chromosome 10, which explained $19 \%$ of the fruit firmness variation in the RIL population (Fig. 1c).

To further investigate the contribution of qFIS1, we crossed two independent recombinant inbred lines, which displayed different phenotypes of CR and shared $90 \%$ sequence identity other than the qFIS1 locus, to construct near-isogenic lines (NILs) by backcrossing for three generations and self-pollinating several times (Supplementary Fig. 1c). The NILs, NIL-FIS1 ${ }^{\mathrm{CC}}$ and NILfis $1^{\mathrm{MM}}$, which were homozygous for the CC or MM $q$ FIS1 alleles, differed at a $55 \mathrm{~kb}$ DNA segment around the locus (Fig. 1d and Supplementary Fig. 1c). We compared CR and skin toughness (ST) between NIL-FIS1 ${ }^{\mathrm{CC}}$ and NIL-fis $1^{\mathrm{MM}}$ and found that the fruit of NIL-fis $1^{\mathrm{MM}}$ could endure higher pressure before bursting and puncturing (Fig. 1d), indicating that qFIS1 contributes to fruit firmness. In line with the fruit firmness, sustained water loss of the NIL-FIS1 ${ }^{\mathrm{CC}}$ fruits was $\sim 2$-fold greater than that of NILfis $1^{\mathrm{MM}}$, and the fruit of NIL-fis $1^{\mathrm{MM}}$ retained good integrity after two weeks of storage (Fig. 1e, f). In addition, the NIL-fis $1^{\mathrm{MM}}$ fruits showed fewer small stained spots than NIL-FIS1 ${ }^{\text {CC }}$ by toluidine blue staining (Supplementary Fig. 1d). These results indicated that mutation of FIS1 results in long shelf life in tomato. Notably, fruit ripening was not influence by qFIS1 (Fig. 1g), and the fruit taste (based on the contents of sugars and acids) also showed no changes in NIL-fis ${ }^{\mathrm{MM}}$ (Fig. 1h). Meanwhile, there was no significant difference in any of the following phenotypes between NIL-FIS1 ${ }^{\mathrm{CC}}$ and NIL-fis ${ }^{\mathrm{MM}}$ : fruit weight, fruit shape, and fruit color (Fig. 1g and Supplementary Fig. 1e). Compared with NIL-FIS1 ${ }^{\mathrm{CC}}$, no alteration of the cell morphology and cell layers of the pericarp that might affect fruit firmness was observed in NIL-fis $1^{\mathrm{MM}}$ (Supplementary Fig. 1f, g). Therefore, these results indicate that qFIS1 specifically controls tomato fruit firmness without negative consequences for other fruit traits and ripening.

FIS1 controls fruit firmness in tomato. To fine-map qFIS1, we conducted high-resolution linkage analysis of 400 individuals of the $\mathrm{BC}_{2} \mathrm{~F}_{3}$ population and 3400 individuals of the $\mathrm{BC}_{3} \mathrm{~F}_{3}$ population. The qFIS1 was finally narrowed to a $17.8 \mathrm{~kb}$ region between markers M8 and M9 (Fig. 2a and Supplementary Table 1), in which two annotated genes, Solyc10g007570 and Solyc10g007580 (Fig. 2a), and a partial promoter region of Solyc10g007550 were located. Unlike the annotated coding sequence of Solyc10g007570, we proved that Solyc10g007570 has a long first exon including two exons and the first intron annotated in the tomato reference genome (ITAG3.0) (Supplementary 
a

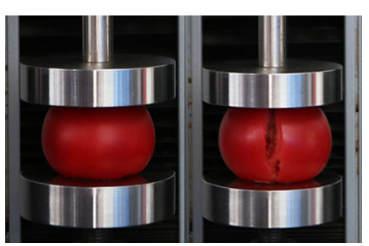

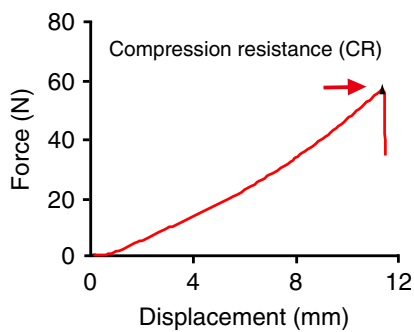

b

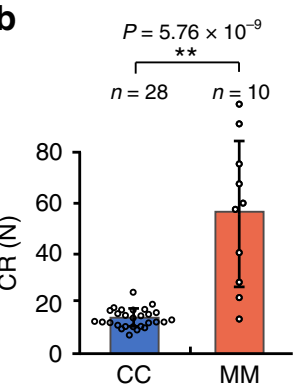

d
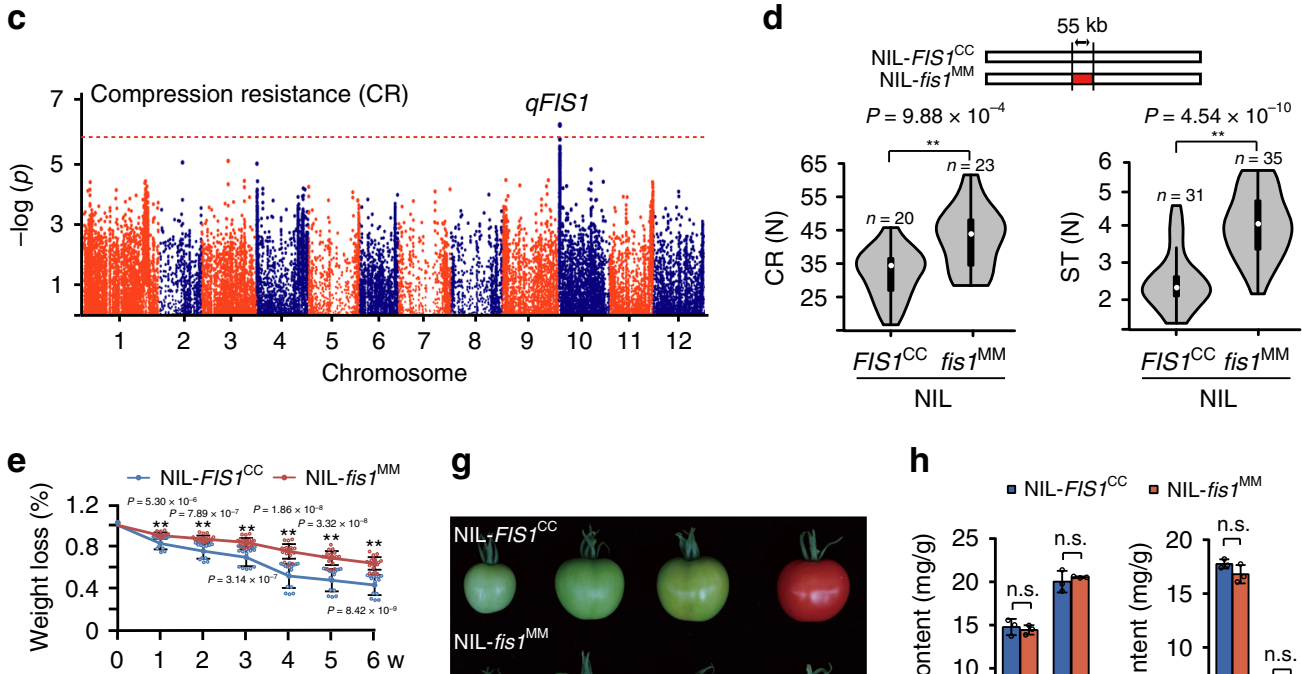

f

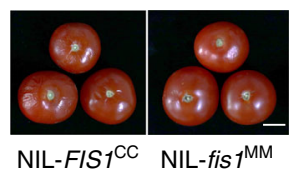

g

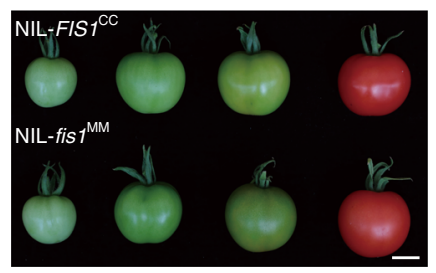

30 DPA $\quad$ MG
BR h

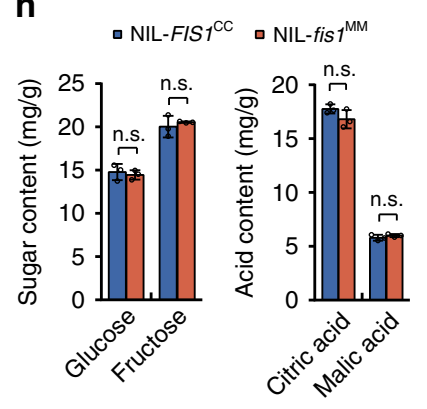

Fig. 1 Identification and characterization of qFIS1. a Photos show the setup for measuring the compression resistance (CR) of tomato red ripe fruits by a texture analyzer. The red arrow indicates the pressure when the fruit is broken. $\mathbf{b} C R$ of two parental lines. Error bars, mean \pm SD. $n=$ fruit number. The asterisks indicate a statistically significant difference (two-tailed Student's $t$-test, ${ }^{\star \star} P<0.01$ ). c Manhattan plots of SNPs associated with CR in the RIL population. The horizontal red line indicates the genome-wide significance threshold $\left(1 \times 10^{-6}\right)$, and the $x$ axis shows the chromosomal position. $\mathbf{d}$ Diagram of the DNA region that differed in the two NILs and violin plots of compression resistance (CR) and skin toughness (ST) for NIL-fis 7 MM and NIL-FIS7CC red ripe fruits. $n=$ fruit number. The asterisks indicate a statistically significant difference (two-tailed Student's $t$-test, ${ }^{\star \star} P<0.01$ ). e Water loss of NIL-fis ${ }^{M M M}$ and NIL-FISTCC red ripe fruits during storage. W, week; Error bars, mean \pm SD. Seventeen fruits for NIL-FIS7CC and 18 fruits for NIL-fis 7 MM were used for this experiment. The asterisks indicate a statistically significant difference (two-tailed Student's $t$-test, ${ }^{\star \star} P<0.01$ ). $\mathbf{f}$ Pictures of NIL-fis 7 MM and NIL-FIS7CC red ripe fruits stored at room temperature for 2 weeks. Bar $=2 \mathrm{~cm}$. $\mathbf{g}$ NIL-fis $7 \mathrm{MM}$ and NIL-FIS7CC fruits at different developmental stages. 30 DPA, 30 days post anthesis; MG, mature green; BR, breaker; RR, red ripe. Bars $=2 \mathrm{~cm}$. h Contents of sugars and acids of NIL-fis ${ }^{\mathrm{MM}}$ and NIL-FIS7 $7 \mathrm{C}$ red ripe fruits. Error bars, mean \pm SD. $n=$ three biological replicates. n.s., no significant difference (two-tailed Student's $t$-test, $P>0.05$ ).

Fig. 2a, b). According to the correct sequence, a single nucleotide insertion in the first exon of Solyc10g007570 in MM led to early termination of translation (Fig. 2a and Supplementary Fig. 2c). In addition, an SNP at the Solyc10g007580 coding region and fifteen SNPs at the Solyc10g007550 promoter region were identified when comparing MM and CC genomic sequences. However, the expression levels of Solyc10g007550 and Solyc10g007570 in fruits were not different between NIL-FIS1 ${ }^{\mathrm{CC}}$ and NIL-fis $1^{\mathrm{MM}}$, while Solyclog007580 was not expressed in fruits (Supplementary Fig. 2d). These results suggested that Solyc10g007570 is a candidate gene for the qFIS1 locus.

To determine the function of Solyc10g007570, we mutated Solyc10g007570 in NIL-FIS1 ${ }^{\text {CC }}$ by CRISPR/Cas9-mediated genome editing. Two null Solyc10g007570 alleles that showed no offtarget mutagenesis on other homologous genes (Supplementary Table 2), named fis $1 \mathrm{cr}-1$ and -2 with a 7 bp deletion and a $14 \mathrm{bp}$ deletion, respectively, were obtained (Fig. 2b). The fruits of fis $1 \mathrm{cr}$ mutants exhibited higher CR than NIL-FIS1 ${ }^{\text {CC }}$ fruits (Fig. 2c). To further verify whether the variation in the coding sequence of Solyc10g007570 was responsible for fruit firmness, we constructed a complementation vector containing the coding sequence (CDS) of CC driven by its native promoter and transformed it into the parental line, MM. Compared with the wild-type MM, the CR and ST were obviously reduced in the transgenic lines (Fig. 2d and Supplementary Fig. 2e, f). These results demonstrate that Solyc10g007570 is FIS1 and that disruption of FIS1 function could enhance tomato fruit firmness.

FIS1 encodes a GA2-oxidase controlling GA catabolism to regulate fruit firmness. FIS1 encodes a GA2-oxidase, a gibberellin deactivating enzyme that catalyzes active GAs into inactive products $^{25}$. In Arabidopsis, the specific-tissue expression patterns of GA2-oxidases help modulate bioactive GAs responsible for many aspects of plant development ${ }^{26}$. According to the 
a

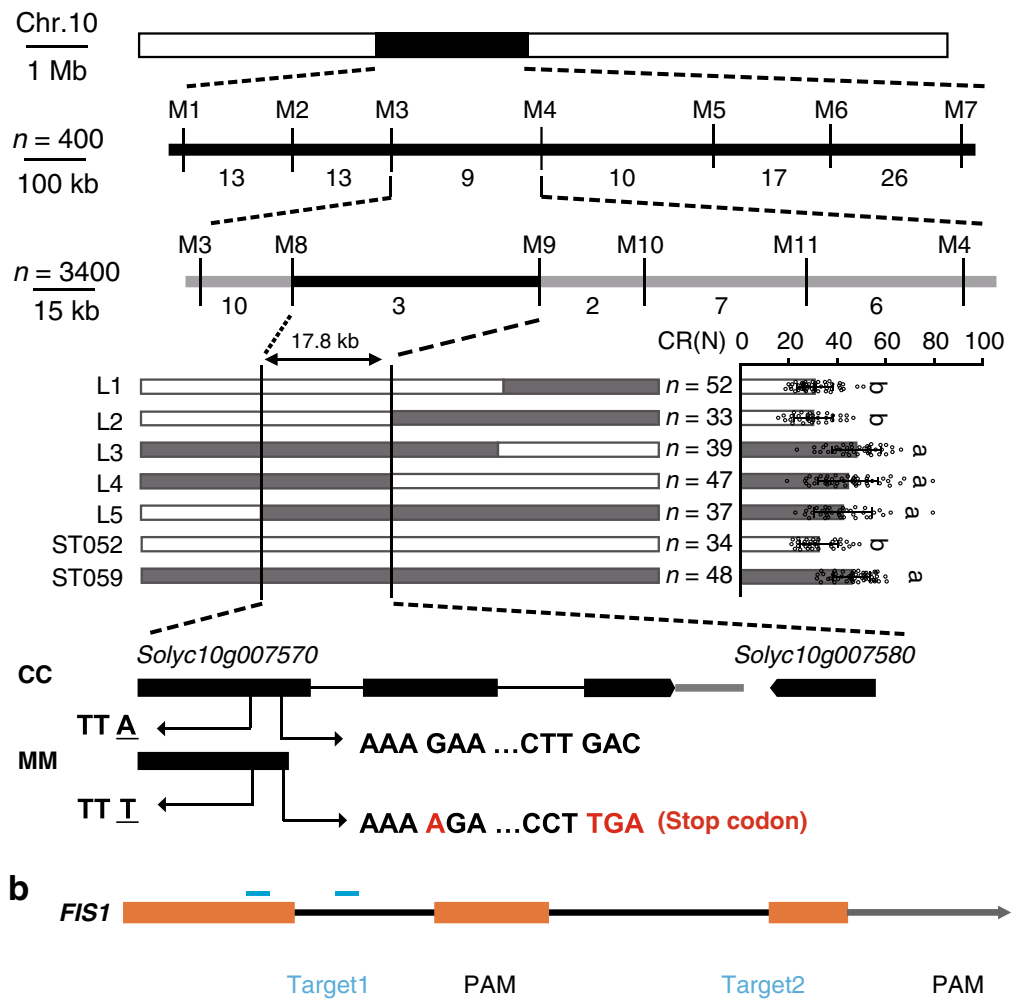

WT ACCCCTTGACCCTTTTGGCTATGGGA. . . TAATTAGACTAGGACCCGTCGAGGT fis1cr-1 ACCCCTTGACC-------CTATGGGA... TAATTAGACTAGGACCCGTCGAGGT fis1cr-2 ACCCCT------------ATGGGA... TAATTAGACTAGGACCCGTCGAGGT

C

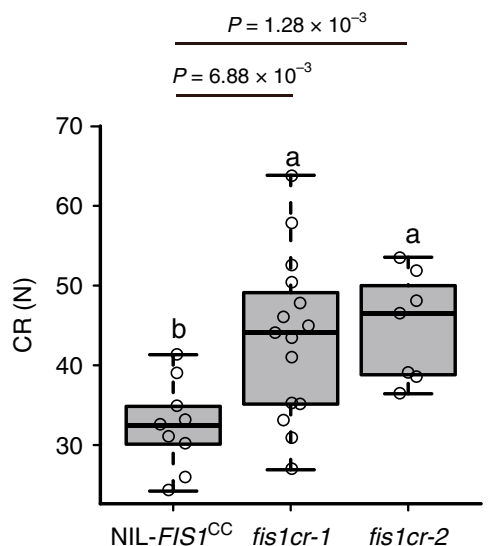

d

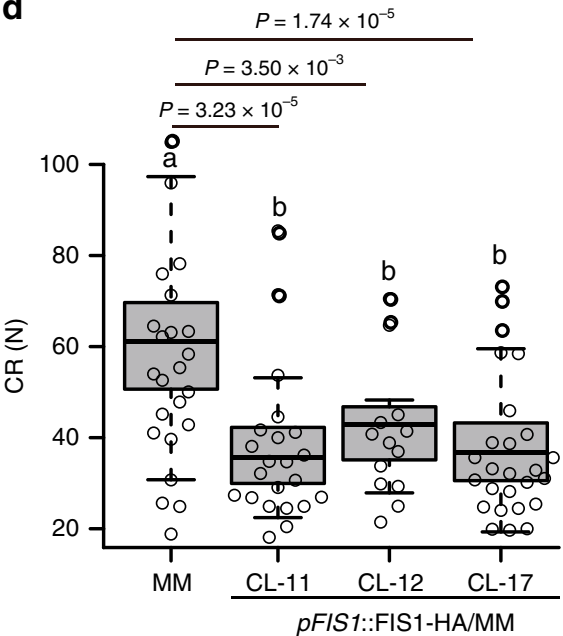

Fig. 2 FIS1 is responsible for fruit firmness formation in tomato. a Fine mapping of qFIS1. Top panel: positional cloning narrowed qFIS1 to the DNA segment between markers M8 and M9. The numbers below the bars indicate the number of recombinants. $n=$ plant number. Middle panel: high-resolution mapping of $q$ FIS1 (left) and CR of recombinant progeny (right). Error bars, mean \pm SD. $n=$ fruit number. Different letters indicate significant differences according to the Tukey-Kramer test $(P<0.05) . P=3.58 \times 10^{-18}$ (L1 and ST059), $P=9.91 \times 10^{-15}$ (L2 and ST059), $P=0.55$ (L3 and ST059), $P=0.28$ (L4 and ST059), $P=0.07$ (L5 and ST059), $P=1.65 \times 10^{-12}$ (ST052 and ST059). Bottom panel, genomic diagram showing the two candidate genes. In addition to one SNP, a single nucleotide insertion in the 1st exon of Solyc10g007570 in MM causes early termination. b FIS1 mutations generated by CRISPR/Cas9 using two single-guide RNAs. Blue lines indicate the target sites of the guide RNAs. Black lines indicate the protospacer-adjacent motif (PAM). The sequences of the two fis 1 mutants in NIL-FIS1CC are shown. c Box plots of red ripe fruit CR for fis $1 \mathrm{Cr}-1 / 2$ mutants and NIL-FIS1CC. $n=9,15$, and 17 fruits. Box edges represent the 0.25 and 0.75 quantiles, and the bold lines indicate median values. Whiskers indicate 1.5 times the interquartile range. Different letters indicate significant differences according to the Tukey-Kramer test $(P<0.05)$. d Box plots of red ripe fruit CR for MM and three transgenic complementation lines (CL-11, CL-12, CL-17). $n=23,22,13$, and 27 fruits. Box edges represent the 0.25 and 0.75 quantiles and the bold lines indicate median values. Whiskers indicate 1.5 times the interquartile range. Different letters indicate significant differences according to the Tukey-Kramer test $(P<$ 0.05). 
a

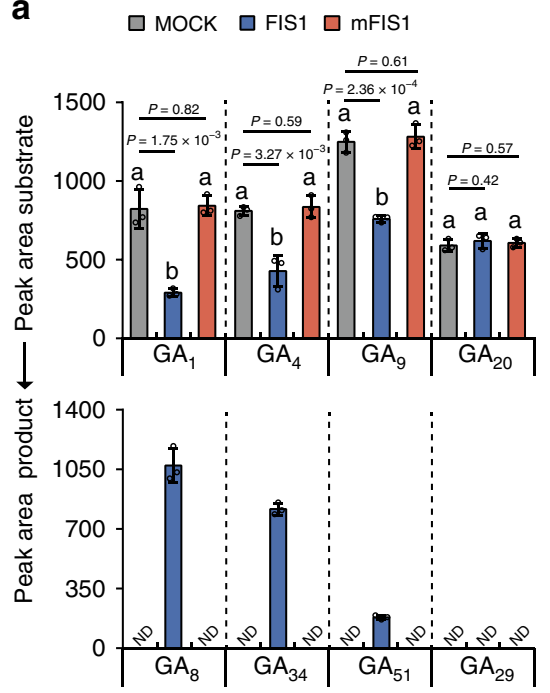

b

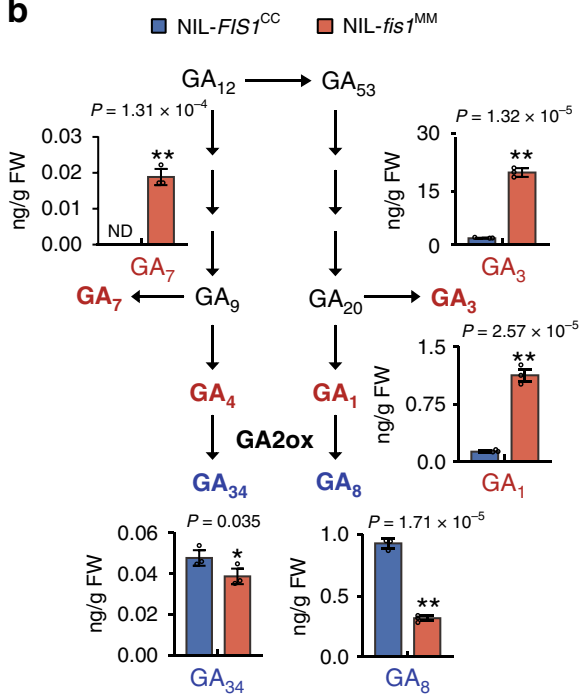

C

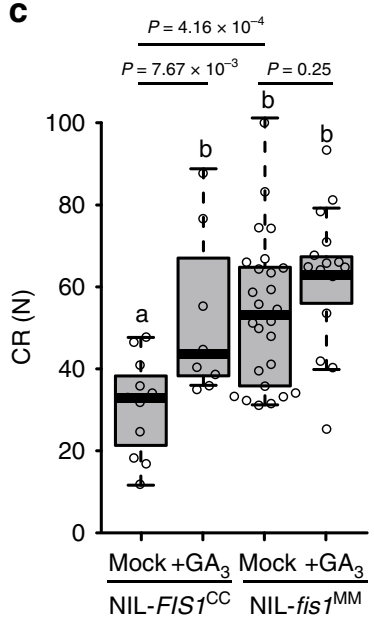

Fig. 3 FIS1 regulates fruit firmness via the GA catabolism mediated pathway. a Enzymatic activities of recombinant FIS1 and mFIS1 proteins. mFIS1,

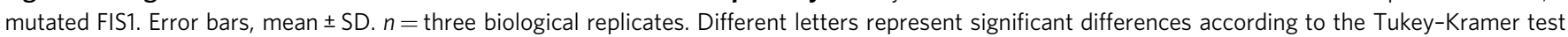
$(P<0.05)$. b The endogenous levels of gibberellins in NIL-fis $7^{\mathrm{MM}}$ and NIL-FIS1CC red ripe fruit pericarp. Error bars, mean \pm SD. $n=$ three biological replicates. The asterisks indicate a statistically significant difference (two-tailed Student's $t$-test, ${ }^{\star} P<0.05$, ${ }^{\star} P<0.01$ ). c $\mathrm{CR}$ of $\mathrm{GA}$-treated or untreated $\mathrm{NIL}$-fis ${ }^{\mathrm{MM}}$ and NIL-FIS1CC red ripe fruits. GA was applied at the mature green stage. $n=11,8,26$, and 15 fruits. Box edges represent the 0.25 and 0.75 quantiles, and bold lines indicate median values. Whiskers indicate 1.5 times the interquartile range. Different letters represent significant differences according to the Tukey-Kramer test $(P<0.05)$.

phylogenetic tree, FIS1 is the sixth member of the GA2-oxidase family and belongs to subgroup I (Supplementary Fig. 3a) ${ }^{27}$. It was highly expressed in tomato fruits from 0 days post anthesis (DPA) to the orange fruit stage, but its transcription was very low in other tissues (Supplementary Fig. 3b). We further analyzed its expression pattern by in situ hybridization and found that FIS1 was mainly expressed in the placenta, locular tissue, parenchyma cells, and seeds of 10 DPA fruits (Supplementary Fig. 3c). These results suggested that FIS1 may play specific roles in regulating fruit development. Actually, except for fruit firmness, NIL-FIS1CC and NIL-fis $1^{\mathrm{MM}}$ showed no effects on fruit and ripening characteristics, as we previously observed (Fig. $1 \mathrm{~g}$ and Supplementary Fig. 1e-g), and no change in plant architecture (Supplementary Fig. 4a). Similarly, compared with MM, fruit weight, color, ripening time, and plant height were not obviously altered in the complementation lines (Supplementary Fig. 4b-d), indicating that FIS1 plays a specific function in controlling fruit firmness in tomato.

GA2-oxidase catalyzes the hydroxylation of the C-2 of active C19-GAs, which includes $\mathrm{GA}_{1}$ and $\mathrm{GA}_{4}$ and their immediate precursors $\mathrm{GA}_{20}$ and $\mathrm{GA}_{9}$, to produce biologically inactive $\mathrm{GAs}^{25}$. To characterize whether FIS1 is a functional GA2-oxidase, we detected its enzyme activity using several $\mathrm{C}_{19}$ and $\mathrm{C}_{20}$ GA substrates. The FIS1 protein was able to convert $\mathrm{GA}_{1}, \mathrm{GA}_{4}$, and $\mathrm{GA}_{9}$ to their corresponding $2 \beta$-hydroxylated products $\mathrm{GA}_{8}, \mathrm{GA}_{34}$, and $\mathrm{GA}_{51}$, respectively, but the mutated FIS1 (mFIS1) was not, indicating that FIS1 is an active enzyme (Fig. 3a). To determine whether the mutation in FIS1 affects endogenous GAs, we measured GA content in the pericarps of red ripe (RR) fruits of NIL-fis $1^{\mathrm{MM}}$ and NIL-FIS1 ${ }^{\mathrm{CC}}$. Bioactive GAs, including $\mathrm{GA}_{1}, \mathrm{GA}_{3}$, and $\mathrm{GA}_{7}$, were dramatically increased in NIL-fis $1^{\mathrm{MM}}$ fruits, whereas the metabolic products of GA2-oxidases, including $\mathrm{GA}_{8}$ and $\mathrm{GA}_{34}$, were decreased in NIL-fis $1^{\mathrm{MM}}$ relative to NIL-FIS1 $\mathrm{CC}$ (Fig. 3b). These differences indicate that disrupted FIS1 activity reduces gibberellin catabolism in the pericarp of tomato fruit. To test whether exogenous GA could enhance the fruit firmness of NIL-FIS1 ${ }^{\text {CC }}$, we sprayed exogenous GA $_{3}$ on NIL-FIS1 ${ }^{\text {CC }}$ mature green fruits and determined the CR of treated and untreated RR fruits. The CR of treated NIL-FIS1 ${ }^{\mathrm{CC}}$ fruits significantly increased compared to the untreated control. Interestingly, exogenous GA treatment slightly increased but did not significantly influence the CR of NIL-fis $1^{\mathrm{MM}}$ fruits (Fig. 3c). Taken together, these results demonstrate that FIS1 functions in GA catabolism to regulate fruit firmness without compromising other plant and fruit characteristics.

FIS1 regulates fruit firmness by influencing cuticle accumulation and deposition. To investigate how FIS1 regulates firmness, we performed RNA-seq using the fruit pericarps of the NILs at 30 DPA and breaker (BR) fruit. A principal component analysis (PCA) showed that the NIL-fis $1^{\mathrm{MM}}$ and NIL-FIS1 ${ }^{\mathrm{CC}}$ samples were located closely in 30 DPA fruits, while they departed in BR fruits (Supplementary Fig. 5a). A total of 938 genes at the 30 DPA stage and 1818 genes at the BR stage showed more than 1.5-fold expression differences between MM and CC NIL alleles (Supplementary Fig. 5b and Supplementary Data 1). Gene ontology (GO) analysis showed that genes involved in metabolic processes and gibberellic acid-mediated signaling pathways were significantly enriched among up- or downregulated genes, respectively (Supplementary Fig. 5c, d). Moreover, genes in the cutin, suberine, and wax biosynthesis pathways were upregulated based on Kyoto Encyclopedia of Genes and Genomes (KEGG) analysis (Supplementary Fig. 5e). Therefore, considerable numbers of genes encoding catalytic enzymes at different steps in cutin and cuticular wax biosynthetic pathways were upregulated in NILfis $1^{\mathrm{MM}}$ at $30 \mathrm{DPA}$ or BR fruits compared to NIL-FIS1 ${ }^{\mathrm{CC}}$ (Fig. 4a). For example, the cytochrome P450 genes, CYP86A69 (Solyc08g081220) and CYP77A-LIKE (Solyc03g119200) participate in cutin biosynthesis ${ }^{28,29}$, GDSLs (Solyc03g111550 and Solyc04g081770) are probably required for extracellular polymerization and cutin deposition ${ }^{30,31}$, and CERs (Solyc07g006680, Solyc01g088400 and Solyc11g067190) required for cuticular wax biosynthesis $^{11}$. The expression levels of these genes were further 
a

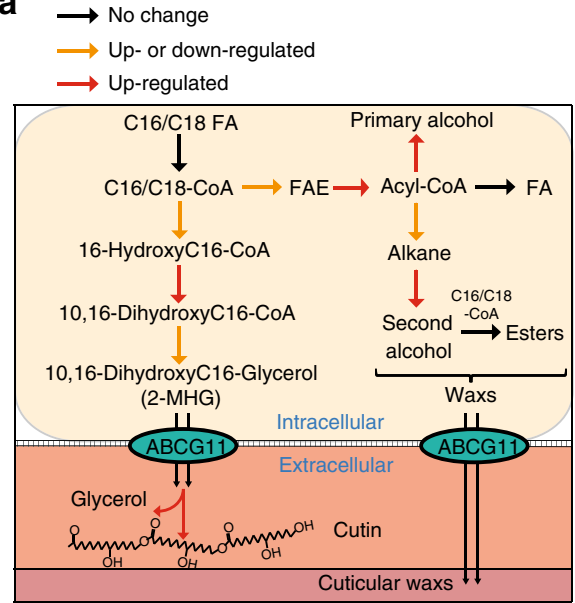

b

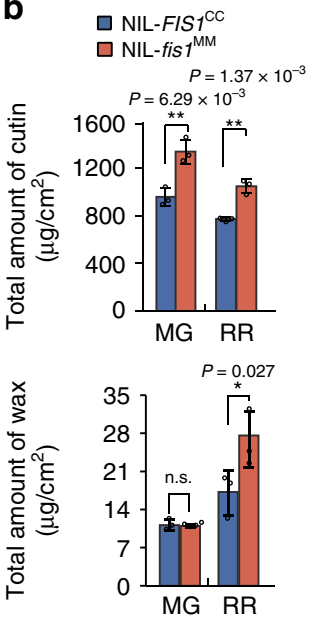

C

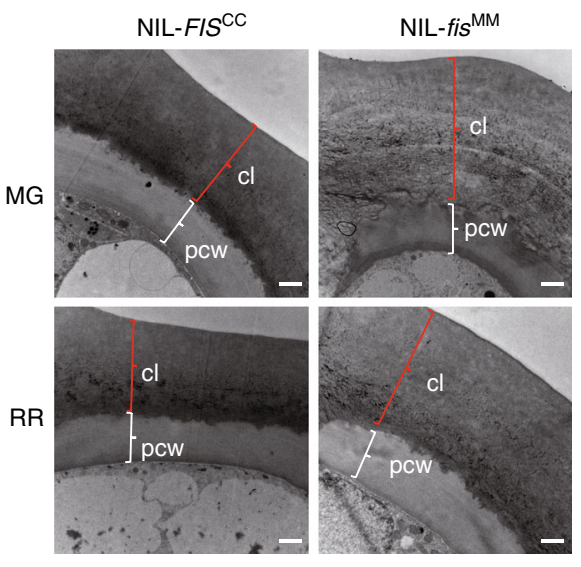

d

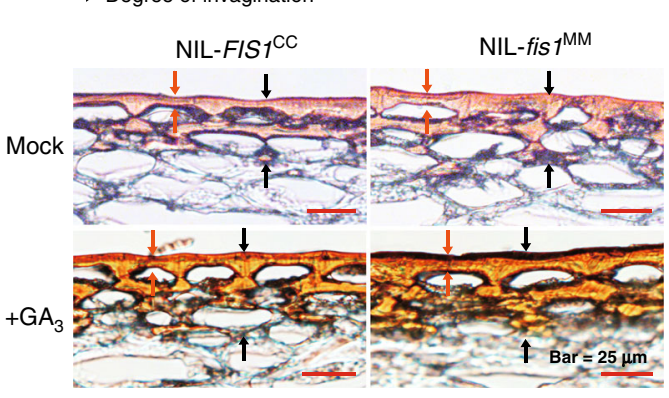

e

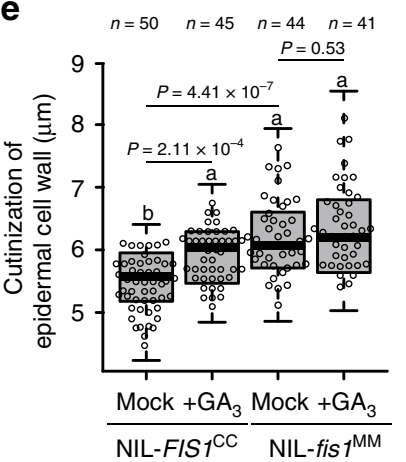

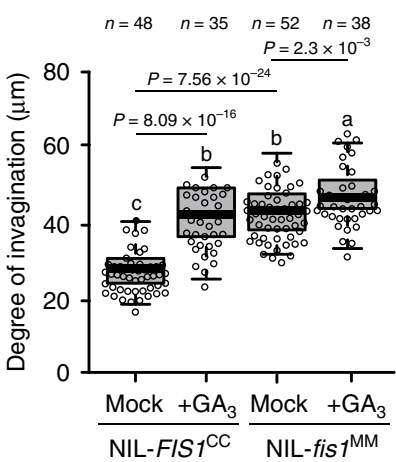

Fig. 4 FIS1 affects cuticle composition and deposition in tomato fruit pericarp. a Diagram of the cuticle biosynthesis pathway. Transcriptome data were obtained for NIL-fis7 MM and NIL-FIS7CC 30 DPA and breaker fruits, and the different colored arrows indicate changes in the expression level of known or putative cuticle-modifying genes that function at the indicated biosynthetic steps. The red arrows indicate that all DEGs related to this step were upregulated in NIL-fis $1 \mathrm{MM}$. Orange arrows indicate that some DEGs related to this step were upregulated in NIL-fis $7 \mathrm{MM}$. The black arrows indicate that the transcript levels of structural genes did not differ between NIL-fis7 $\mathrm{MM}^{\mathrm{M}}$ and NIL-FIS7 CC. $\mathbf{b}$ Total cutin and wax content in the fruit pericarps of NIL-fis7MM and NIL-FIS7CC. The asterisks indicate a statistically significant difference. Error bars, mean \pm SD. $n=$ three biological replicates. The asterisks indicate a statistically significant difference (two-tailed Student's $t$-test, ${ }^{\star} P<0.05,{ }^{* \star} P<0.01$ ). n.s., no significant difference (two-tailed Student's $t$-test, $P>0.05$ ). MG, mature green; RR, red ripe. c Transmission electron microscopy images of NIL-fis $7^{M M}$ and NIL-FIS7CC fruit pericarp sections. Bar $=2 \mu \mathrm{m}$. MG, mature green; RR, red ripe. CL, cuticle layer; PCW, polysaccharide cell wall. Three independent experiments were performed. $\mathbf{d}$ Cuticle sections stained with Sudan IV to visualize the cutinization of epidermal cell walls and the degree of invagination with or without gibberellin treatment in NIL-fis7MM and NIL-FIS7CC. Bars $=25 \mu \mathrm{m}$. Six independent experiments were performed. e Quantification of the cutinization of epidermal cell walls and the degree of invagination at the red ripe fruit after GA treatment in NIL-fis $7^{\mathrm{MM}}$ and NIL-FIS7CC. $n=$ section number. Box edges represent the 0.25 and 0.75 quantiles, and the bold lines indicate median values. Whiskers indicate 1.5 times the interquartile range. Different letters indicate significant differences according to the Tukey-Kramer test $(P<0.05)$.

analyzed by reverse-transcription quantitative real-time PCR (QPCR) in the pericarps of two NILs. Obvious increases in their transcripts were observed in NIL-fis $1^{\mathrm{MM}}$ consistent with RNAseq (Supplementary Fig. 6 and Supplementary Data 1). These results suggest that FIS1 might control cuticle biosynthesis of fruit to influence fruit firmness 9 .

To test whether the biosynthesis of cutin and cuticular wax in the pericarp was affected by the variation in FIS1, we measured the cuticle components in NIL-fis $1^{\mathrm{MM}}$ and NIL-FIS1 ${ }^{\mathrm{CC}}$. The contents of total cutin (Fig. 4b) and some monomers, including $10,16-\mathrm{DiOH}$ hexadecanoic (2-MHG), which is the main monomer, were significantly increased by $\sim 30 \%$ in both mature green and red ripe fruits of NIL-fis $1^{\mathrm{MM}}$ compared to that of NILFIS1 ${ }^{\text {CC }}$ (Supplementary Table 3). In addition, the total wax amount was also higher in NIL-fis $1^{\mathrm{MM}}$ than in NIL-FIS1 $1^{\mathrm{CC}} \mathrm{RR}$ fruits, and two main classes of wax, alcohols, and alkanes, showed nearly $82 \%$ and $67 \%$ increases in NIL-fis $1^{\mathrm{MM}}$, respectively (Fig. $4 \mathrm{~b}$ and Supplementary Table 3). Moreover, we investigated the thickness of the cuticle including the cutinization of epidermal cell walls and the degree of invagination that affects the cuticle properties by histological staining ${ }^{32}$. The characteristics of cutinization of the epidermal cell wall and the degree of invagination clearly revealed cuticle thickening in mature green and red ripe fruits of NIL-fis $1^{\mathrm{MM}}$ (Supplementary Fig. 7a, b). Consistent with this, a dramatic increase in cuticle thickness in NIL-fis $1^{\mathrm{MM}}$ was also observed by transmission electron microscopy (TEM). However, no obvious difference in the ultrastructure of the epidermal cell wall was observed (Fig. 4c and Supplementary Fig. 7c). As we previously observed, GA treatment could enhance fruit firmness in NIL-FIS1 ${ }^{\mathrm{CC}}$. We also measured the contents of cutin and wax and analyzed the cuticle morphology of NIL-FIS1 ${ }^{\text {CC }}$ after GA treatment. The total cutin content of RR fruits was significantly enhanced in NIL-FIS1 CC but not in NIL-fis $1^{\mathrm{MM}}$ after GA treatment compared with untreated fruits (Supplementary Fig. 7d). In contrast, the wax amount was increased in GA-treated fruits of both NIL-FIS1 CC 
and NIL-fis ${ }^{\mathrm{MM}}$ compared to the untreated fruits (Supplementary Fig. 7d). In accordance with the increases in cutin and wax contents, epidermal cell cutinization and epidermal invagination were also significantly thickened in NIL-FIS1 ${ }^{\mathrm{CC}}$ after GA treatment (Fig. 4d, e). When we complemented the MM with FIS1, the cuticle thickness of transgenic RR fruits became thinner than that of MM fruits (Supplementary Fig. 7e, f). Collectively, these data indicate that FIS1 regulates fruit firmness by influencing cuticle accumulation and deposition in tomato fruits.

Selection of FIS1 enhances tomato fruit firmness during domestication. Fruit firmness is a key target for cultivar selection in tomato breeding. Analysis of FIS1 variation in 166 tomato varieties and 53 S. pimpinellifolium accessions $s^{33}$ indicated that the FIS1 locus was under selection during tomato domestication (Fig. 5a). Moreover, FIS1 is located in a previously reported selective sweep ${ }^{33}$. Approximately $97 \%$ of the cultivated tomatoes and $57 \%$ of the early domesticated cherry tomatoes were homozygous for fis $1^{\mathrm{MM}}$ alleles, whereas all of the analyzed wild species were homozygous for $F I S 1^{\mathrm{CC}}$ except the heterozygous alleles (Fig. 5b), indicating that the FIS1 allele was positively selected during tomato domestication. To determine the contribution of FIS1 in domesticated tomatoes, we quantified the effect of allelic variation at FIS1 on tomato fruit firmness in 30 tomato accessions collected from different regions worldwide (Supplementary Table 4). We found that tomato accessions with the fis $1^{\mathrm{MM}}$ genotype had higher CR than tomato species with the FIS1 ${ }^{\mathrm{CC}}$ genotype (Fig. 5c). Thus, FIS1 is a target selected in tomato breeding for fruit firmness.

Genome editing of FIS1 improves tomato fruit firmness. Disruption of FIS1 function could increase tomato fruit firmness with negligible effects on other fruit traits. Therefore, FIS1 could be a very useful target to improve fruit firmness in tomato breeding. To validate this hypothesis, we mutated FIS1 in two tomato accessions by CRISPR/Cas9-mediated genome editing. One of them belongs to wild S. pimpinellifolium named PP, and the other is a cherry tomato cultivar, TS20 $5^{33}$. Two independent fislcr mutants were obtained in the PP or TS205 background, which were named $\mathrm{PP} /$ fis 1 cr-1 and PP/fis 1 cr-2 with 2 and $1 \mathrm{bp}$ deletions (Fig. 5d), respectively, and TS205/fislcr-1 and TS205/ fislcr-2 with 8 and 7 bp deletions, respectively (Fig. 5e). As expected, all of these fislcr mutants exhibited higher CR than their respective controls (Fig. 5f, g). With the increase in fruit firmness, the cuticles of RR fruits were significantly thickened in these fis1cr mutants compared with PP or TS205 (Supplementary Fig. 8a-c). At the same time, the fruit weights showed no obvious difference (Supplementary Fig. 8d, e). These results confirm that FIS1 is an ideal target for the improvement of fruit firmness in tomato.

\section{Discussion}

During the process of tomato domestication, not only did fruit yield improve significantly but also fruit texture changed greatly compared with its progenitors. Our present findings demonstrate the following causal sequence of changes related to selection at the FIS1 locus during tomato domestication: disrupted FIS1 function due to a 1-bp frameshift insertion, increased accumulation of bioactive GAs in the fruit pericarp, enhanced cutin biosynthesis and cuticle accumulation, and increased tomato fruit firmness with negligible effects on other phenotypes (Supplementary Fig. 8f).

Fruit firmness is an important trait that affects consumer acceptance, fruit transportability, and shelf life. Nevertheless, fruit firmness is a highly complex trait that is determined by a number of factors involving numerous pathways. Extensive studies on fruit firmness have focused on the remodeling and degradation of the cell wall ${ }^{5,8}$. In addition, cuticle properties have also been suggested to contribute to fruit firmness. The fruit cuticle is a continuous hydrophobic structure composed predominantly of two components: cutin and wax ${ }^{11}$. Dynamic changes in cutin and wax compositions during fruit development are associated with cuticle accumulation that could affect fruit firmness in a direct way by acting as a supporting barrier under tension, or indirectly regulating fruit water status 7,9 . The mutated and knocked-down genes involved in cuticle biosynthesis often result in a thinner cuticle layer, leading to a decrease in fruit firmness $7,9,30$. In our study, the cuticle thickness (Supplementary Fig. 7a, b) and cutin and wax contents of the NIL-fis $1^{\mathrm{MM}}$ fruits (Supplementary Table 3) were strikingly increased compared to those of CC allele fruits that is the cause of the enhanced fruit firmness in MM allele. In contrast, the CR of mature green fruits displayed no significant differences between NIL-fis ${ }^{\mathrm{MM}}$ and NIL-FISI ${ }^{\mathrm{CC}}$, but an obvious increase in ST could be observed in NIL-fis $1^{\mathrm{MM}}$ (Supplementary Fig. 9). One possibility is that the mature green fruits are hard and difficult to break. However, the skin toughness reflects the change in the cuticle layer at the mature green stage. Moreover, cuticle as a protective skin is highly correlated with fruit water loss and shelf life ${ }^{9,10}$. Downregulated cutin/wax contents lead to reduced water retention capacity in fruits ${ }^{10}$. Similarly, the increase in cutin/wax contents in NIL-fis $1^{\mathrm{MM}}$ causes fruits to have a long shelf life (Fig. 1e). Thus, the change in fruit cuticle is the reason for the alteration of fruit firmness caused by the variation in FIS1.

Gibberellin is an important hormone that controls fruit development. Changes in GA catabolism in transgenic tomato and the application of exogenous GA could affect fruit ripening ${ }^{21}$. As a GA2ox protein, FIS1 is a critical catabolic enzyme that maintains endogenous GA levels by catalyzing bioactive GA into bioinactive $\mathrm{GA}^{26}$. Mutation of this gene in NIL-fis $1^{\mathrm{MM}}$ causes an increase in bioactive GA and results in an enhancement of fruit firmness in tomato (Fig. 3b) without significant effects on fruit ripening and morphology. Our work reveals GA may play a role in regulating fruit firmness during fruit ripening. However, FIS1 was mainly expressed in the mesocarp instead of the exocarp during fruit development according to our in situ results (Supplementary Fig. 3c) and tomato laser microdissection RNA-seq data $^{34}$. It is difficult to explain how FIS1 could specifically affect the cuticle layer of tomato fruit exocarp. Actually, GAs can be transported from synthetic tissue into other tissues to perform their functions ${ }^{35}$. One possibility is that more bioactive GAs are transported into exocarp in fis1 mutants. Moreover, FIS1 and the other five GA2oxs display redundant and specific functions in tomato fruit development. Our results and those from tomato fruit RNA-seq datasets indicate that four GA2ox genes are expressed in fruits but with different spatial-temporal expression patterns, especially Solyc07g056670 (Supplementary Fig. 10). This latter gene is highly expressed during ripening in both the tomato inner and outer epidermis ${ }^{34}$. Therefore, revealing the functions of Solyc07g056670 in GA metabolism and fruit development will help us to address this question.

Bioactive GAs induce the transcription of cutin and wax synthetic genes in many species ${ }^{36,37}$. Our RNA-seq assay also indicated that a dozen genes related to cutin and wax biosynthesis are upregulated with the accumulation of bioactive GA in NILfis ${ }^{\mathrm{MM}}$ (Supplementary Data 1). These upregulated genes were required for the biosynthesis and extracellular polymerization of cuticle $^{30}$ that determine fruit viscoelastic behavior and fruit firmness ${ }^{7,9}$. Therefore, our results suggest that FIS1 manipulates cuticle biosynthetic processes to regulate fruit firmness. Previous 


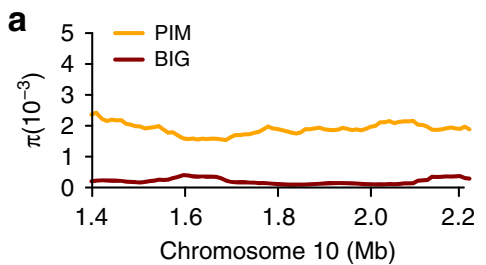

b
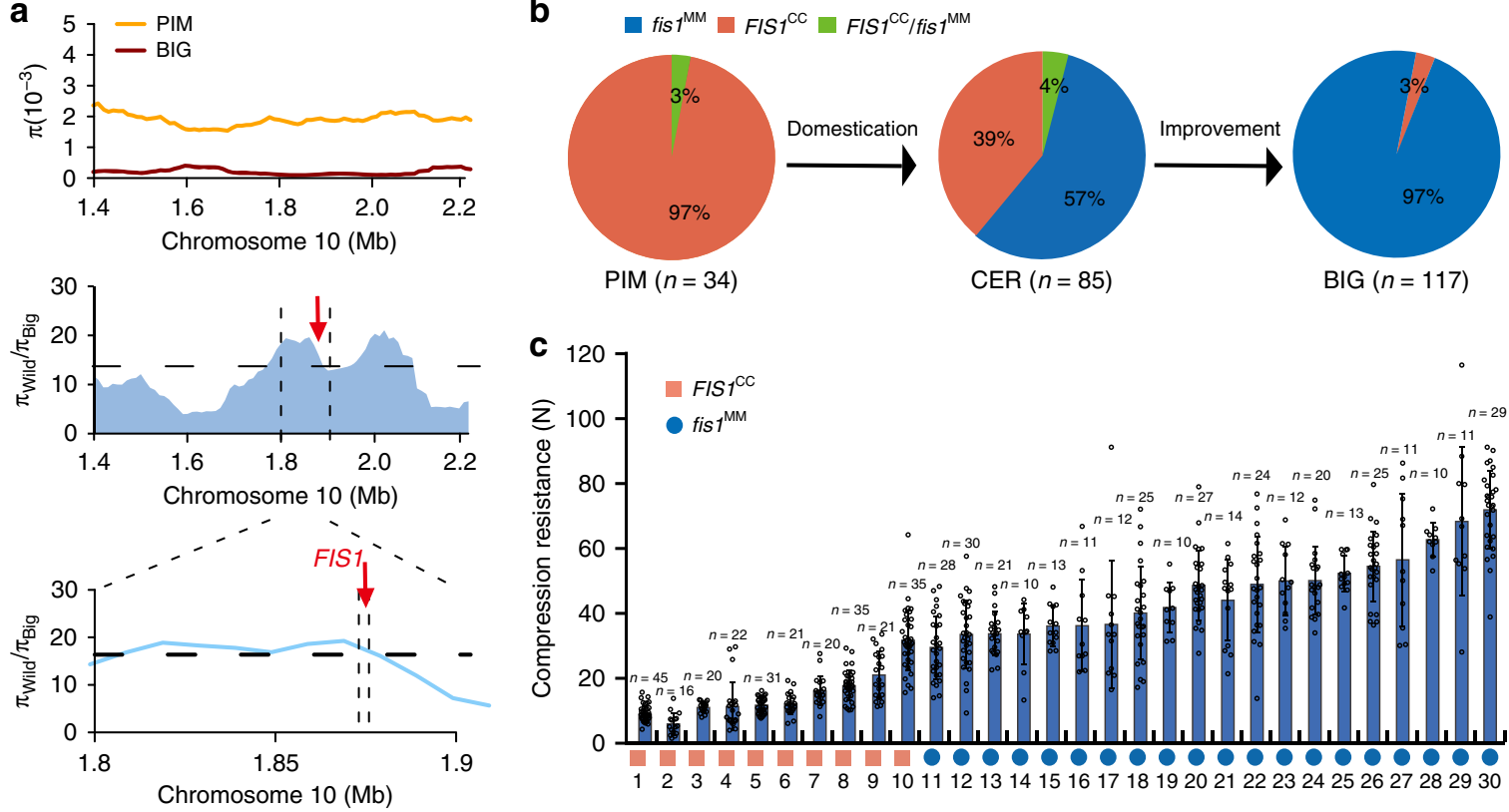

d

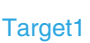

PAM

Target2

PAM

WT ACCCCTTGACCCTTTTGGCTATGGGA... СTTAATTAGACTAGGACCCGTCGAGGT

PP/fis1cr-1 ACCCCTTGAC--TTTTGGCTATGGGA. . СTTAATTAGACTAGGACCCGTCGAGGT

PP/fis1cr-2 ACCCCTTGACCCTTTTGG-TATGGGA... СTTAATTAGACTAGGACCCGTCGAGGT

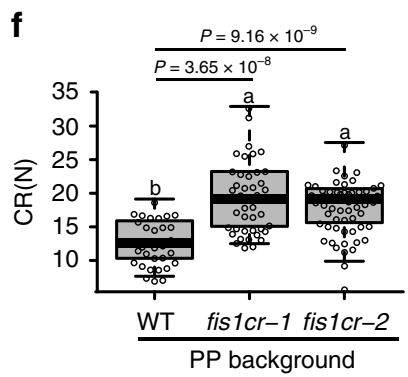

e

$\begin{array}{cccc}\text { Target1 } & \text { PAM } & \text { Target2 } & \text { PAM } \\ \text { WT ACCCCTTGACCCTTTTGGCTATGGGA } & \ldots \text { CTTAATTAGACTAGGACCCGTCGAGGT }\end{array}$

TS205/fis1Cr-1 ACCCCTTGACC--------TATGGGA. . CTTAATTAGACTAGGACCCGTCGAGGT

TS205/fis1cr-2 ACCCCTTGACCC-------TATGGGA... CTTAATTAGACTAGGACCCGTCGAGGT

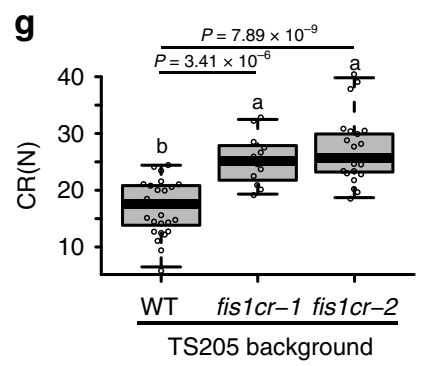

Fig. 5 FIS1 is selected during tomato domestication and is a target for the improvement of fruit firmness. a Scan for selection signature surrounding the FIS1 gene. Top: nucleotide diversity $(\pi)$ of wild species (orange line) and big-fruit cultivars (red line). Middle: $\pi$ ratio of the wild species to big-fruit cultivars (blue region) for a region on chromosome 10. The horizontal dashed lines indicate the genome-wide top 5\% ratio cutoff for candidate domestication sweeps. The vertical black dashed lines (bottom) delimit FIS1. b Frequency distribution of the FIS1 genotype in natural populations including 236 resequenced tomato accessions. PIM: Solanum pimpinellifolium, CER: S. lycopersicum var. cerasiforme, BIG: Big-fruit tomatoes. c CR of 20 cultivated tomatoes with the fis $7 \mathrm{MM}$ allele and 10 cherry tomatoes with the FIS7 $\mathrm{CC}$ allele. Error bars, mean \pm SD. $n=$ fruit number. $\mathbf{d}$ FIS1 mutations generated by CRISPR-Cas9 using two single-guide RNAs in PP. Blue lines indicate the target sites of the guide RNAs. Black lines indicate the protospacer-adjacent motif (PAM). The sequences of PP/fis1cr-1/2 mutants are shown. e FIS1 mutations generated by CRISPR/Cas9 using two single-guide RNAs in TS205. The sequences of TS205/fis 1 cr-1/2 mutants are shown. $\mathbf{f}$ Box plots of red ripe fruit CR for PP/fis $1 \mathrm{cr}-1 / 2$ mutants and PP. $n=31,42$, and 58 fruits. Box edges represent the 0.25 and 0.75 quantiles, and the bold lines indicate median values. Whiskers indicate 1.5 times the interquartile range. Different letters indicate significant differences according to the Tukey-Kramer test $(P<0.05)$. $\mathbf{g}$ Box plots of red ripe fruit CR for TS205/fis $1 \mathrm{Cr}-1 / 2$ mutants and TS205. $n=28,12$, and 23 fruits. Box edges represent the 0.25 and 0.75 quantiles and the bold lines indicate median values. Whiskers indicate 1.5 times the interquartile range. Different letters indicate significant differences according to the Tukey-Kramer test $(P<0.05)$.

studies have revealed that SHINES (SHNs) act in cuticle accumulation by regulating the expression levels of several downstream genes associated with cuticle biosynthesis in Arabidopsis and tomato ${ }^{28,36}$. Although we found that SlSHN2 displayed a higher expression level in NIL-fis $1^{\mathrm{MM}}$ than in NIL-FIS1 $1^{\mathrm{CC}}$, whether SlSHN2 is one of the transcription factors that participates in the FIS1-mediated cuticle biosynthetic pathway still needs to be explored in the future.

Fruit firmness is always the main target in tomato breeding. Ripening mutants have been used as parents to generate $F_{1}$ hybrids in order to confer firm fruit and long shelf life ${ }^{3,4}$. However, the hybrids also inherited poor traits, such as bad flavor and 
poor color ${ }^{4}$. Moreover, cell-wall-modifying related genes have been used as targets for obtaining hard fruit. Except for pectate lyase $(P L)^{5}$, silencing most of these genes yielded slight or even undetectable effects on fruit firmness 8,38 . Therefore, few genes could be used to improve fruit firmness to date. Using the CRISPR-edited method generates fislcr mutants in tomato accessions that could improve fruit firmness effectively without taking unfavorable changes in fruit quality (Fig. $5 \mathrm{~d}-\mathrm{g}$ and Supplementary Fig. 8d, e). Thus FIS1 is a useful target to enhance fruit firmness in tomato. Taken together, our work provides new insights into the biological mechanism underlying fruit firmness and raises the prospect of improving fruit hardness.

\section{Methods}

Plant materials and growth conditions. RILs containing 219 individual lines were derived from S. lycopersicum cv. Moneymaker (MM) and S. lycopersicum var. cerasiforme (CC) were used as materials for association analysis, as we described previously ${ }^{24}$. To clone the gene, we generated a $\mathrm{BC}_{3} \mathrm{~F}_{2}$ population derived from the cross between two RILs, ST052 and ST059, which shared 90\% sequence identity other than the qFIS1 locus. Cultivated tomato MM, NIL-FIS1 CC, PP (S. pimpinellifolium), and cherry tomato TS205 were used for the transgenic experiments. Field experiments were performed in a greenhouse at the Nankou experimental station in Beijing, China. Seedlings were grown in a commercial nursery for $30-40$ days and then transplanted to fields.

Phenotypic evaluation. CR and ST were measured using an Instron 5542 texture analyzer according to a previously reported method ${ }^{23}$. Eight red ripe fruits of each plant and four plants of each line were used for CR and ST measurements.

More than ten plants of each genotype were used to evaluate fruit weight. The average fruit weight of each plant was represented by the average fruit weight of all fruits on the second inflorescence. The average fruit weight of ten plants represented the fruit weight of each genotype.

For cell layer and cell area measurements, 10-15 fruits of each NIL line were picked at the mature green stage. Five free-hand sections from the equator of each fruit were used to analyze the average cell area by staining with $0.1 \%$ toluidine blue for $10 \mathrm{~s}$. Images were taken with a Leica DFC450C microscope. The cell layer was measured in the abaxial-adaxial direction of the pericarp. The average cell area was measured in two $(\alpha$ and $\beta$ ) regions and was calculated using the following formula: average cell area $=$ area $(\alpha+\beta) /$ total cell number $(\alpha+\beta)$.

Content analysis of sugars and acids. More than 8 red ripe fruits were collected from each NIL line for sugar and acid analysis. The mixed fruit pericarp was ground in liquid nitrogen, and then $100 \mathrm{mg}$ of ground powder was diluted in $2 \mathrm{ml}$ water. The following was added to the water-soluble tomato pericarp extracts: saccharide internal standard $(1 \mathrm{mg} / \mathrm{ml}$ arabinose water) and acid internal standard $(0.5 \mathrm{mg} / \mathrm{ml}$ lactic acid). After sonication and centrifugation, the samples were filtered through a $0.22 \mu \mathrm{m}$ polyethersulfone ultrafiltration membrane and mixed with an equal volume of acetonitrile for analysis. Saccharide and acid contents were measured by UPLC-MS/MS (ACQUITY UPLC I-Class-Xevo TQ-S Micro, Waters). For saccharide analysis, an ACQUITY UPLC BEH Amide $1.7 \mu \mathrm{m}$ column was used as the analytical column $(2.1 \times 100 \mathrm{~mm}$; Waters $)$. The mobile phase was composed of acetonitrile as solvent $\mathrm{A}$ and $1 \mathrm{mg} / \mathrm{ml}$ ammonium hydroxide as solvent $\mathrm{B}$. The temperatures of the column and autosampler were $60^{\circ} \mathrm{C}$ and $4{ }^{\circ} \mathrm{C}$, respectively. Each saccharide was separated by increasing the solvent $\mathrm{B}$ concentration from $10 \%$ to $20 \%$ over $6 \mathrm{~min}$ after the first $2 \mathrm{~min}$ of the run at $10 \%$ using a flow rate of $0.2 \mathrm{ml} / \mathrm{min}$, followed by washing with $10 \%$ solvent B for $2 \mathrm{~min}$. For acid analysis, an ACQUITY UPLC HSS T3 $1.8 \mu \mathrm{m}$ column was used as the analytical column $(2.1 \times 100 \mathrm{~mm}$; Waters). The mobile phase was composed of acetonitrile containing $1 \mathrm{mg} / \mathrm{ml}$ formic acid as solvent A and water containing $1 \mathrm{mg} / \mathrm{ml}$ formic acid as solvent B. The temperatures of the column and autosampler were $25^{\circ} \mathrm{C}$ and $4{ }^{\circ} \mathrm{C}$, respectively. The acid elution was performed at a flow rate of $0.1 \mathrm{ml} / \mathrm{min}$ with $90 \%$ B for $5 \mathrm{~min}$. Data analysis was performed using MassLynx V4.1 (Waters).

Water loss and cuticle permeability measurements. A total of 10-15 fruits of each NIL line were picked at the RR stage and then stored at room temperature for 6 weeks. Fruit weight was measured every week. Water loss was calculated as the percentage of the decrease in fruit weight. For measurements of cuticle permeability, MG fruits of NILs were collected and dropped in 1\% toluidine blue solution staining for $12 \mathrm{~h}$ as described in Hovav et al. ${ }^{39}$.

Associated analysis and gene mapping. The genome sequencing data of the RIL population and their parents were obtained from our previously published work ${ }^{24}$. The paired-end reads of MM, CC, and the RILs were mapped to the tomato reference genome (SL2.50). SNP calling was performed on the alignment results using the Genome Analysis Toolkit (GATK) version 3.1.1 and Picard package version $1.119^{40}$. The calling was performed according to the following steps:
(1) unmapped reads were deleted, (2) duplicate reads were deleted, (3) alignment using the IndelRealigner package in GATK was conducted, and then (4) SNP calling for each sample was performed using the UnifiedGenotyper package in GATK, with a minimum base quality score of 20 . To ensure the quality of SNP calling in MM and CC, SNPs were filtered further with the VariantFiltration package in GATK using parameters $\mathrm{QD}<2.0\|\mathrm{FS}>60.0\| \mathrm{MQ}<40.0 \|$ MQRankSum $<-12.5 \|$ ReadPosRankSum $<-8.0$, and SNPs with DP $<10$ were also removed. The remaining SNPs between MM and CC were used as the index for SNP calling in the 219 RILs. The SNPs in the 219 RILs were filtered with the Lowqual tag marked by the GATK UnifiedGenotyper package. To infer the missing genotype at an SNP site of an individual line, 20 SNPs flanking the target SNP in other lines of the population were compared with the individual line. If all of the lines having the same genotype for the flanking SNPs as the individual line had the same genotype at the target SNP, this SNP genotype was inferred/imputed to the individual line. The RIL association study was conducted using the imputed information for the SNPs of the 219 RILs by the compressed mixed linear model implemented in GAPIT ${ }^{41}$. The cutoff $P$ value was set as $1 \mathrm{E}-6$. For fine mapping, based on the genotypes of $400 \mathrm{BC}_{3} \mathrm{~F}_{2}$ individuals derived from the cross of ST052 and ST059, two recombinant lines of the RILs and 22 recombinant plants were obtained for the progeny test and the FIS1 gene was mapped between markers M3 and M4. An additional 28 recombinant plants were screened from $3400 \mathrm{BC}_{3} \mathrm{~F}_{3}$ individuals by genotyping with six markers, including M3, M4, M8, M9, M10, and M11 (Supplementary Table 1). Together with the progeny test results, we delimited $q F I S 1$ to a $17.8 \mathrm{~kb}$ region between the Indel markers M8 and M9. The markers used for mapping are listed in Supplementary Table 1.

Construction design and plant transformation. For CRISPR-Cas 9 constructs, two gRNAs, target1 and target2, were designed using the CRISPR-P v2.0 tool (http://cbi.hzau.edu.cn/CRISPR2/). A pair of primers, P1, containing two sgRNAs and AarI recognition sites was used to amplify the Target1_U6-26t_SlU6p_Target2 fragments using the pCBC_DT1T2_SlU6p vector as the template, which was revised from the pCBC_DT1T2 vector ${ }^{42}$ by replacing AtU6p with $S I U 6 p^{43}$. Then, the PCR product was digested with AarI to generate the gRNA cassette, and inserted into pCAMBIA2300_35 S_Cas9_SIU6p_sgRNA for CRISPR/Cas9 construction, which was modified from pCAMBIA2300 by inserting a SlU6p_sgRNA after the NOS terminator and a rice optimized Cas $9^{44}$ after the $35 \mathrm{~S}$ promoter. The complementary construct contained a 2420-bp promoter region of the FIS1 gene and a 1008-bp coding sequence, which were amplified from CC using the primers P4 and P5, respectively. The DNA fragment was inserted into the pCAMBIA2300HA vector to generate the construct pFIS1::FIS1-HA. CRISPR and complementary plasmids were introduced into Agrobacterium tumefaciens AGL1 competent cells and transformed into NIL-FIS1 CC, PP, TS205, and MM by Agrobacterium-mediated transformation. The transgenic lines were confirmed by PCR and sequencing All experiments were performed using homozygous lines from the $T_{2}$ generation without T-DNA integration. The PCR primers used for plasmid construct and transgenic line determination are listed in Supplementary Table 1. The off-target sites were predicted using the online tool (http://crispr.mit.edu), and then the sequences of targets were amplified using genomic DNA of transgenic plants as template and then analyzed by sequencing.

Phylogenetic trees and motif recognition. The amino-acid sequences of GA2oxs of Arabidopsis and tomato were downloaded from the NCBI database, and aligned using ClustalW. A phylogenetic reconstruction analysis was performed based on the neighbor-joining method using PHYML version 3.0 under the JTT evolution model. The reliability of the obtained trees was tested by bootstrapping with 500 replicates. Motif analysis was performed using the EMBL-EBI database (http:// smart.embl-heidelberg.de/). Multiple-sequence alignments were performed with a gap open penalty of 10 and gap extension penalty of 5 using DNAMAN 6.0 and ClustalW $2.0^{45}$

In situ hybridization. In situ hybridization was performed as described in Scott et al. with modification ${ }^{46}$. Cubes of $\sim 5$-mm per side tissue from NIL-FIS $1^{\mathrm{CC}} 10$ DPA fruits were fixed for $24 \mathrm{~h}$ at $4{ }^{\circ} \mathrm{C}$ in freshly prepared $4 \%$ (w/v) paraformaldehyde buffered with phosphate-buffered saline (PBS, pH 7.2). Fixed tissues were dehydrated in a graded ethanol:Histochoice (H2779-1L, Sigma) series and impregnated with Paraplast (P3683-1kg, Sigma). Dewaxed thin sections $(10 \mu \mathrm{m})$ were hybridized with the hydrolysis probes at a concentration of $2 \mathrm{ng} / \mu \mathrm{l}$ in hybridization buffer for $12 \mathrm{~h}$ at $55^{\circ} \mathrm{C}$. Hybridization buffer for each slide: $20 \mu \mathrm{l}$ of $10 \times$ buffer ( $3 \mathrm{M} \mathrm{NaCl}, 0.1 \mathrm{M}$ Tris- $\mathrm{HCl}$ pH 6.8, $0.1 \mathrm{M} \mathrm{NaPO}_{4}, 50 \mathrm{mM}$ EDTA), $40 \mu \mathrm{l}$ of $50 \%$ dextran sulfate (S4030, Merck-Millipore), $80 \mu \mathrm{l}$ of deionized formamide (0606-100mL, Amresco), $4 \mu \mathrm{l}$ of 50× Denhardt's solution (30915-25mL, Sigma), $39 \mu \mathrm{l}$ of formamide (V900064-500 mL, Sigma), and $2 \mu \mathrm{l}$ of tRNA (10109541001, Roche) in $200 \mu$ of DEPC- $\mathrm{H}_{2} \mathrm{O}$. Color development complete sections were observed using a fluorescence microscope (DM5500, Leica).

For the FIS1 probe, the FIS1 coding region was amplified with the primers P16: $5^{\prime}$-GCATCTATTTTGGGTCTCAATCCA- $3^{\prime}$ and $5^{\prime}$ -

CATCAGGACATGGAGGATAATG- ${ }^{\prime}$, and cloned into pEAZY-T3 (TransGen), which contained T7 and SP6 promoter sequences. M13F and M13R (N53002, Thermo Fisher) were used to amplify recombinant fragment containing cDNA 
from the FIS1 and T7 and SP6 promoters. In vitro transcription was performed with T7 RNA polymerase using the purified PCR product as the template to generate the antisense or sense probe.

Cuticle staining. Fruits were collected for each NIL line at mature green and red ripe fruit stages. The pericarp equator was cut into $2-\mathrm{mm}$ cubes for fixation in FAA buffer (water:formaldehyde:glacial acetic acid:ethanol 1:2:10:7). The fixed samples were dehydrated in a graded series of ethanol $(70,85,95$, and $100 \%)$, followed by a xylene/ethanol series (xylene/ethanol 1:3, 1:1, 3:1, and 100\% xylene). Xylene was replaced gradually using a paraffin/xylene series (paraffin/xylene 1:3, 1:1, and 3:1) at $37^{\circ} \mathrm{C}, 45^{\circ} \mathrm{C}$, and $60^{\circ} \mathrm{C}$ for $12 \mathrm{~h}$, respectively. Finally, the sample solution was replaced with fresh paraffin every $12 \mathrm{~h}$ for four times. A Leica RM2235 microtome was used to cut $10 \mu \mathrm{m}$ sections for staining with $0.05 \%$ Sudan IV. Cuticle thickness was calculated using ImageJ.

Transmission electron microscopy (TEM). For the TEM assay, a total of 6-8 mature green or red ripe fruits of each NIL line were used. Cubes of $\sim 2$-mm per side were excised from the fruit equator. Methods of sample fixation, embedding, and slicing were performed as described with modification ${ }^{12}$. Samples were fixed in primary fixative containing $2.5 \%(\mathrm{v} / \mathrm{v})$ glutaraldehyde in $0.05 \mathrm{M}$ phosphate buffer (pH 6.8) for $4-6 \mathrm{~h}$ at room temperature. Samples were washed with phosphate buffer and postfixed in $1 \%(\mathrm{v} / \mathrm{v})$ osmium tetroxide and $1.5 \%$ potassium ferricyanide. Fixed samples were washed with water and then dehydrated through a gradient ethanol series (50, 70, 80, 90, and 100\%). Prepared samples were infiltrated with propylene oxide resin mixtures and polymerized in $100 \%$ resin for $48 \mathrm{~h}$ at $60^{\circ} \mathrm{C}$. Ultrathin sections $(80-100 \mathrm{~nm})$ were stained with $2 \%$ uranyl acetate and Reynold's lead citrate and viewed with a H-7500 (Hitachi) transmission electron microscope. Images were analyzed using ImageJ software.

Content analysis of cutin monomer and wax. Cutin monomer analysis was performed according to a previously described method with modification ${ }^{9}$. Isolated peels $\left(10-\mathrm{cm}^{2}\right.$ area) were incubated at $85^{\circ} \mathrm{C}$ in 2 -propanol with $0.01 \%(\mathrm{w} / \mathrm{v}) 2,6$-ditert-butyl-4-methylphenol for $30 \mathrm{~min}$, and then the solution was replaced with fresh 2-propanol. After $2 \mathrm{~h}$, the cuticles were washed in a chloroform/methanol series (chloroform/methanol 2:1, 1:1, and 1:2), for $12 \mathrm{~h}$ at each step. After washing with methanol to remove the chloroform, the cuticles were depolymerized in $8 \mathrm{ml}$ of anhydrous methanol containing 7.5\% (v/v) methyl acetate and 4.5\% (w/v) sodium methoxide at $60^{\circ} \mathrm{C}$. Methyl heptadecanoate and v-pentadecalactone were added as internal standards. After $2 \mathrm{~h}$, the reaction was cooled and $1 \mathrm{ml}$ of acetic acid was added to adjust $\mathrm{pH}$, followed by two dichloromethane extractions $(10 \mathrm{ml})$ to remove methyl ester monomers. The organic phase was washed three times with $0.9 \% \mathrm{NaCl}(\mathrm{w} / \mathrm{v})$, dried with 2,2-dimethoxypropane, and dried under nitrogen gas. The residue was prepared for GC by derivatization using N,O,-bis(trimethylsilyl)trifluoroacetamide (BSTFA). The samples were dried and then dissolved in $\mathrm{n}-$ hexane:toluene (1:1) prior to analysis on an Agilent GC-MS instrument (7890B5977A, Agilent) with $30 \mathrm{~m} \times 0.25 \mathrm{~mm}$ HP-5 MS columns and helium as the carrier gas. GC was performed with temperature-programmed automatic injection at $60^{\circ} \mathrm{C}$, holding for $5 \mathrm{~min}$ at $60^{\circ} \mathrm{C}$, temperature increase to $230^{\circ} \mathrm{C}$ at a rate of $2{ }^{\circ} \mathrm{C}$ $\mathrm{min}^{-1}$, and holding for $40 \mathrm{~min}$ at $230^{\circ} \mathrm{C}$.

The cuticular wax analysis was modified from a previously reported method ${ }^{9}$. The chloroform-soluble cuticular wax extracts from tomato pericarp of a known area $\left(100 \mathrm{~cm}^{2}\right)$ were evaporated to dryness under a stream of nitrogen gas, and the dried residue was prepared for GC by derivatization using N,O,-bis(trimethylsilyl)trifluoroacetamide (BSTFA). The experiment was carried out with temperatureprogrammed automatic injection at $70^{\circ} \mathrm{C}$, held for $5 \mathrm{~min}$ at $70^{\circ} \mathrm{C}$, raised by $2{ }^{\circ} \mathrm{C}$ $\min ^{-1}$ to $180^{\circ} \mathrm{C}$, and held for $5 \mathrm{~min}$ at $180^{\circ} \mathrm{C}$.

Enzyme activity of recombinant FIS1 protein. In vitro protein expression was performed according to previously described method with minor modifications ${ }^{47}$. The full-length cDNAs of FIS1 amplified from MM and CC were cloned into the pIX-HALO (ABRC) vector using the primer pair, P17 (Supplementary Table 1). The $p I X-H A L O$ vector was used as a control. Confirmed constructs were used for recombinant protein expression in wheat germ TNT expression systems (L4140, Promega). For the enzyme activity assay, the procedure was adapted from previous studies with slight modification ${ }^{48,49}$. Fifty or seventy-seven microliters of total protein was incubated with GA metabolites ( $10 \mathrm{ng}$ of $\mathrm{GA}_{1}, 2 \mathrm{ng}$ of $\mathrm{GA}_{4}, 2 \mathrm{ng}$ of $\mathrm{GA}_{9}, 2 \mathrm{ng}$ of $\mathrm{GA}_{20}$, OlChemIm) in $100 \mu \mathrm{l}$ of reaction buffer containing $100 \mathrm{mM}$ Tris- $\mathrm{HCl}$ (pH 7.5), $1 \mathrm{mM} \mathrm{FeSO}_{4}, 10 \mathrm{mM}$ 2-oxoglutarate, $10 \mathrm{mM}$ ascorbate, and $5 \mathrm{mM} \mathrm{DTT}$ at $30^{\circ} \mathrm{C}$ for $6 \mathrm{~h}$. After incubation, $100 \mathrm{ml}$ of methanol was added and mixed. After centrifugation, the supernatant was collected for analysis by LC-MS/ MS (ACQUITY UPLC I-Class-Xevo TQ-S Micro, waters) with an ACQUITY UPLC BEH C18 $(2.1 \mathrm{~mm} \times 50 \mathrm{~mm}, 1.7 \mu \mathrm{m})$ column according to a method modified from previous studies ${ }^{50}$. Acetonitrile was mobile phase $\mathrm{A}$ and water with $0.05 \%$ formic acid was mobile phase B. The run was at a flow rate of $0.2 \mathrm{ml} / \mathrm{min}$ with an initial $3 \%$ of solvent A. Solvent A changed from 3\% to $65 \%$ in $17 \mathrm{~min}$, then from 65 to $90 \%$ in $1.5 \mathrm{~min}$ and was held at $90 \%$ for $1 \mathrm{~min}$; it was returned to $3 \%$ from $90 \%$ in $1.5 \mathrm{~min}$. Finally, solvent A was held at $3 \%$ for $1.5 \mathrm{~min}$. The column and injection room (FTN) temperatures were all $20^{\circ} \mathrm{C}$. The injection volume was $1 \mu \mathrm{l}$ for all samples. Mass was set in negative ion mode for GA analysis. The ESI source parameters were set as follows: electrospray capillary voltage, $3.0 \mathrm{kV}$; source temperature, $150^{\circ} \mathrm{C}$; desolvation temperature, $600^{\circ} \mathrm{C}$; desolvation gas flow, $650 \mathrm{~L} / \mathrm{h}$; cone gas flow, $10 \mathrm{~L} / \mathrm{h}$. The mass acquisition was performed in multiple reaction monitoring for $22.5 \mathrm{~min}$.

Content analysis of endogenous GAs. Pericarps of 10-15 red ripe fruits of the NIL lines were used for GA content measurement, as described in previous work, with minor modifications ${ }^{51}$. Five hundred milligrams of the ground plant powder was extracted with $90 \%$ aqueous methanol $(\mathrm{MeOH})$. As internal standards for $\mathrm{GA}$ content measurement, 2 ng of each D-labeled GA compound was added to the extracting solvents. The MAX cartridge (Waters Corporation, Milford, USA) was activated and equilibrated with $\mathrm{MeOH}$, water, $5 \% \mathrm{NH}_{4} \mathrm{OH}$, and $90 \% \mathrm{MeOH}$, and MCX (Waters Corporation, Milford, USA) was activated with $\mathrm{MeOH}$, water, and $90 \% \mathrm{MeOH}$. The crude extracts were loaded onto the tandem cartridges connected with an adapter. The MAX cartridge was disconnected, rinsed with $5 \% \mathrm{NH}_{4} \mathrm{OH}$ in $5 \% \mathrm{MeOH}$, and then rinsed with $\mathrm{MeOH}$. GA compounds were eluted with $90 \%$ $\mathrm{MeOH}$ containing $2 \% \mathrm{FA}$. The eluent was dried under a nitrogen stream and redissolved in $40 \% \mathrm{MeOH}$ prior to UPLC-MS/MS analysis. GA analysis was performed on a quadrupole linear ion trap hybrid mass spectrometer (QTRAP 6500 AB) equipped with an electrospray ionization (ESI) source coupled with a UPLC (Waters). The UPLC inlet method and ESI source parameters were set as in a previous report ${ }^{51}$. GAs were detected in negative multiple reaction monitoring (MRM) mode.

Exogenous $\mathbf{G A}_{\mathbf{3}}$ treatment. The fruits of NILs were marked on the flower opening day and treated with $2.5 \mathrm{mM} \mathrm{GA}_{3}(63492-1 \mathrm{G}$, Sigma-Aldrich) by spraying from the MG stage. All fruits were treated every 3 days, for four times total. Fruits were collected at the RR stage for measurement of fruit weight, fruit compression resistance, and cuticle. Water treatment was the control.

RNA-seq analysis. The RNA-seq reads were aligned to the tomato genome (ITAG2.4) by STAR v2.5.352, and their features were counted by feature Counts v 1.5. $3^{53}$, as described in a previous paper ${ }^{54}$. Using the statistical package DEGseq in $\mathrm{R}$ version 3.0.3, the MA-plot-based method was used to calculate $P$ values that were adjusted using the Benjamini-Hochberg procedure. The fold change between two libraries was calculated as FPKM (fragments per kilobase of transcript sequence per million base pairs sequenced). The thresholds for the identification of differentially expressed genes (DEGs) were as follows: FPKM $>1$ in any tissue, fold change $>1.5$ or $<0.666$, and Benjamini-Hochberg adjusted $P$ value $<0.05$. The primers used for DEG verification are listed in Supplementary Table 1. GO and KEGG analyses of DEGs were performed using their best homologous genes in Arabidopsis with DAVID (The Database for Annotation, Visualization, and Integrated Discovery, https://david.ncifcrf.gov/).

Identification of selective sweeps. To identify the selection region around FIS1, the SNPs near the FIS1 locus (Chr10:1.4-2.2 Mb, SL2.50) in the tomato genome were obtained. We measured the level of nucleotide diversity $(\pi)$ using a $100-\mathrm{kb}$ window with a step size of $10 \mathrm{~kb}$ in wild species (wild) and big-fruit cultivars (big). The ratios of nucleotide diversity between wild and big ( $\pi$ wild/ $\pi \mathrm{big}$ ) were also calculated. According to the strategy described in a previous study ${ }^{33}$, the top $5 \%$ of ratios were used as the cutoff for sweeps.

Reporting summary. Further information on research design is available in the Nature Research Reporting Summary linked to this article.

\section{Data availability}

The RNA sequencing datasets generated in this study and genomic sequencing datasets of RILs have been deposited in the Sequence Read Archive (SRA) under the accession number PRJNA559455 and SRP093370. Other data supporting our findings are available in the manuscript file or from the corresponding author upon request. Source data are provided with this paper.

Received: 6 April 2020; Accepted: 26 October 2020; Published online: 17 November 2020

\section{References}

1. Vincent, $\mathrm{H}$. et al. A prioritized crop wild relative inventory to help underpin global food security. Biol. Conserv. 167, 265-275 (2013).

2. Brummell, D. A. \& Harpster, M. H. Cell wall metabolism in fruit softening and quality and its manipulation in transgenic plants. Plant Mol. Biol. 47, 311-340 (2001)

3. Vrebalov, J. et al. A MADS-box gene necessary for fruit ripening at the tomato ripening-inhibitor (rin) locus. Science 296, 343-346 (2002). 
4. Kitagawa, M. et al. Characterization of tomato fruit ripening and analysis of gene expression in F-1 hybrids of the ripening inhibitor (rin) mutant. Physiol. Plant. 123, 331-338 (2005).

5. Uluisik, S. et al. Genetic improvement of tomato by targeted control of fruit softening. Nat. Biotechnol. 34, 950-952 (2016).

6. Chaib, J. et al. Physiological relationships among physical, sensory, and morphological attributes of texture in tomato fruits. J. Exp. Bot. 58, 1915-1925 (2007).

7. Romero, P. \& Rose, J. K. C. A relationship between tomato fruit softening, cuticle properties and water availability. Food Chem. 295, 300-310 (2019).

8. Smith, C. J. S. et al. Antisense RNA inhibition of polygalacturonase gene expression in transgenic tomatoes. Nature 334, 724-726 (1988).

9. Saladie, M. et al. A reevaluation of the key factors that influence tomato fruit softening and integrity. Plant Physiol. 144, 1012-1028 (2007)

10. Leide, J., Hildebrandt, U., Reussing, K., Riederer, M. \& Vogg, G. The developmental pattern of tomato fruit wax accumulation and its impact on cuticular transpiration barrier properties: effects of a deficiency in a betaketoacyl-coenzyme A synthase (LeCER6). Plant Physiol. 144, 1667-1679 (2007).

11. Yeats, T. H. \& Rose, J. K. The formation and function of plant cuticles. Plant Physiol. 163, 5-20 (2013).

12. Isaacson, $T$. et al. Cutin deficiency in the tomato fruit cuticle consistently affects resistance to microbial infection and biomechanical properties, but not transpirational water loss. Plant J. 60, 363-377 (2009).

13. Tanksley, S. D. et al. Advanced backcross QTL analysis in a cross between an elite processing line of tomato and its wild relative L. pimpinellifolium. Theor. Appl. Genet. 92, 213-224 (1996).

14. Frary, A. et al. Fine mapping of quantitative trait loci for improved fruit characteristics from Lycopersicon chmielewskii chromosome 1. Genome 46, 235-243 (2003).

15. Chapman, N. H. et al. High-resolution mapping of a fruit firmness-related quantitative trait locus in tomato reveals epistatic interactions associated with a complex combinatorial locus. Plant Physiol. 159, 1644-1657 (2012).

16. Vrebalov, J. et al. Fleshy fruit expansion and ripening are regulated by the Tomato SHATTERPROOF gene TAGL1. Plant Cell 21, 3041-3062 (2009).

17. Gimenez, E. et al. Functional analysis of the Arlequin mutant corroborates the essential role of the Arlequin/TAGL1 gene during reproductive development of tomato. PLoS ONE 5, e14427 (2010).

18. Gimenez, E. et al. Transcriptional activity of the MADS Box ARLEQUIN/ TOMATO AGAMOUS-LIKE1 Gene is required for cuticle development of tomato fruit. Plant Physiol. 168, 1036-1048 (2015).

19. Serrani, J. C., Sanjuan, R., Ruiz-Rivero, O., Fos, M. \& Garcia-Martinez, J. L. Gibberellin regulation of fruit set and growth in tomato. Plant Physiol. 145, 246-257 (2007).

20. Garcia-Hurtado, N. et al. The characterization of transgenic tomato overexpressing gibberellin 20-oxidase reveals induction of parthenocarpic fruit growth, higher yield, and alteration of the gibberellin biosynthetic pathway. J. Exp. Bot. 63, 5803-5813 (2012).

21. $\mathrm{Li}, \mathrm{H}$. et al. Gibberellins play a role in regulating tomato fruit ripening. Plant Cell Physiol. 60, 1619-1629 (2019).

22. Martinez-Bello, L., Moritz, T. \& Lopez-Diaz, I. Silencing C19-GA 2-oxidases induces parthenocarpic development and inhibits lateral branching in tomato plants. J. Exp. Bot. 66, 5897-5910 (2015)

23. Jackman, R. L. et al. Measurement of tomato fruit firmness. Hortscience 7 , 781-783 (1990).

24. Zhang, S. et al. Enhancer-Promoter interaction of SELF PRUNING $5 G$ shapes photoperiod adaptation. Plant Physiol. 178, 1631-1642 (2018).

25. Yamaguchi, S. Gibberellin metabolism and its regulation. Annu. Rev. Plant Biol. 59, 225-251 (2008).

26. Hedden, P. \& Phillips, A. L. Gibberellin metabolism: new insights revealed by the genes. Trends Plant Sci. 5, 523-530 (2000).

27. Chen, $S$. et al. Identification and characterization of tomato gibberellin 2oxidases (GA2oxs) and effects of fruit-specific SlGA2ox1 overexpression on fruit and seed growth and development. Hortic. Res. 3, 16059 (2016).

28. Shi, J. X. et al. The tomato SISHINE3 transcription factor regulates fruit cuticle formation and epidermal patterning. N. Phytol. 197, 468-480 (2013).

29. Lashbrooke, J. et al. The tomato MIXTA-Like transcription factor coordinates fruit epidermis conical cell development and cuticular lipid biosynthesis and assembly. Plant Physiol. 169, 2553-2571 (2015).

30. Yeats, T. H. et al. The identification of cutin synthase: formation of the plant polyester cutin. Nat. Chem. Biol. 8, 609-611 (2012).

31. Yeats, T. H. et al. Tomato Cutin Deficient 1 (CD1) and putative orthologs comprise an ancient family of cutin synthase-like (CUS) proteins that are conserved among land plants. Plant J. 77, 667-675 (2014).

32. Petit, J. et al. The glycerol-3-phosphate acyltransferase GPAT6 from tomato plays a central role in fruit cutin biosynthesis. Plant Physiol. 171, 894-913 (2016)
33. Lin, T. et al. Genomic analyses provide insights into the history of tomato breeding. Nat. Genet. 46, 1220-1226 (2014)

34. Martin, L. B. et al. Laser microdissection of tomato fruit cell and tissue types for transcriptome profiling. Nat. Protoc. 11, 2376-2388 (2016).

35. Binenbaum, J., Weinstain, R. \& Shani, E. Gibberellin localization and transport in plants. Trends Plant Sci. 23, 410-421 (2018).

36. Shi, J. X. et al. SHINE transcription factors act redundantly to pattern the archetypal surface of Arabidopsis flower organs. PLoS Genet. 7, e1001388 (2011).

37. Fogelman, E., Stern, R. A. \& Ginzberg, I. Benzyladenine and gibberellin treatment of developing "Pink Lady" apples results in mature fruits with a thicker cuticle comprising clusters of epidermal cells. Protoplasma 252, 1009-1017 (2015)

38. Tieman, D. M., Harriman, R. W., Ramamohan, G. \& Handa, A. K. An antisense pectin methylesterase gene alters pectin chemistry and soluble solids in tomato fruit. Plant Cell 4, 667-679 (1992).

39. Hovav, R., Chehanovsky, N., Moy, M., Jetter, R. \& Schaffer, A. A. The identification of a gene (Cwp1), silenced during Solanum evolution, which causes cuticle microfissuring and dehydration when expressed in tomato fruit. Plant J. 52, 627-639 (2007).

40. McKenna, A. et al. The Genome Analysis Toolkit: a MapReduce framework for analyzing next-generation DNA sequencing data. Genome Res. 20, 1297-1303 (2010)

41. Lipka, A. E. et al. GAPIT: genome association and prediction integrated tool Bioinformatics 28, 2397-2399 (2012).

42. Xing, H. L. et al. A CRISPR/Cas9 toolkit for multiplex genome editing in plants. BMC Plant Biol. 14, 327 (2014).

43. Deng, L. et al. Efficient generation of pink-fruited tomatoes using CRISPR/ Cas9 system. J. Genet. Genomics 45, 51-54 (2018).

44. Shan, Q. et al. Targeted genome modification of crop plants using a CRISPR Cas system. Nat. Biotechnol. 31, 686-688 (2013).

45. Larkin, M. A. et al. Clustal W and Clustal X version 2.0. Bioinformatics 23, 2947-2948 (2007).

46. Harding, S. A., Leshkevich, J., Chiang, V. L. \& Tsai, C. J. Differential substrate inhibition couples kinetically distinct 4-coumarate: coenzyme A ligases with spatially distinct metabolic roles in quaking aspen. Plant Physiol. 128, 428-438 (2002).

47. Bartlett, A. et al. Mapping genome-wide transcription-factor binding sites using DAP-seq. Nat. Protoc. 12, 1659-1672 (2017).

48. Liu, C. et al. Shortened basal internodes encodes a gibberellin 2-oxidase and contributes to lodging resistance in rice. Mol. Plant 11, 288-299 (2018).

49. Sakamoto, T. et al. Expression of a gibberellin 2-oxidase gene around the shoot apex is related to phase transition in rice. Plant Physiol. 125, 1508-1516 (2001).

50. Xin, P. Y. et al. A tailored high-efficiency sample pretreatment method for simultaneous quantification of 10 classes of known endogenous phytohormones. Plant Commun. 1, 1-10 (2020).

51. He, J. et al. CYP72A enzymes catalyse 13-hydrolyzation of gibberellins. Nat. Plants 5, 1057-1065 (2019).

52. Dobin, A. et al. STAR: ultrafast universal RNA-seq aligner. Bioinformatics 29, 15-21 (2013).

53. Liao, Y., Smyth, G. K. \& Shi, W. featureCounts: an efficient general purpose program for assigning sequence reads to genomic features. Bioinformatics $\mathbf{3 0}$ 923-930 (2014)

54. Zhang, S. et al. Spatiotemporal transcriptome provides insights into early fruit development of tomato (Solanum lycopersicum). Sci. Rep. 6, 23173 (2016).

\section{Acknowledgements}

The RIL populations were provided by $\mathrm{J}$. Li, and the S. pimpinellifolium and big-fruit tomato populations were provided by S. Huang. This work was supported by The National Natural Science Foundation of China (31991182 and 31972431), The National Key Research and Development Program of China (2016YFD0100506), the Central Public-interest Scientific Institution Basal Research Fund (Y2020PT29), and the Science and Technology Innovation Program of the Chinese Academy of Agricultural Sciences.

\section{Author contributions}

R.L. and S.S. carried out most of the experiments; H.W. and Z.Z. helped to analyze the cutin content; J.C. and P.X. measured GA content; T.Z. helped to observe the phenotypes of the RIL population; K.W. and Y.H. analyzed the data; H.W. provided technical help with cell-wall analysis and revised the paper. X.C. and J.L. conceived the project, designed the research, and wrote the manuscript.

\section{Competing interests}

The authors declare no competing interests. 


\section{Additional information}

Supplementary information is available for this paper at https://doi.org/10.1038/s41467020-19705-w.

Correspondence and requests for materials should be addressed to X.C.

Peer review information Nature Communications thanks Christophe Rothan and the other, anonymous reviewer(s) for their contribution to the peer review of this work.

Reprints and permission information is available at http://www.nature.com/reprints

Publisher's note Springer Nature remains neutral with regard to jurisdictional claims in published maps and institutional affiliations. (c) (i) Open Access This article is licensed under a Creative Commons Attribution 4.0 International License, which permits use, sharing, adaptation, distribution and reproduction in any medium or format, as long as you give appropriate credit to the original author(s) and the source, provide a link to the Creative Commons license, and indicate if changes were made. The images or other third party material in this article are included in the article's Creative Commons license, unless indicated otherwise in a credit line to the material. If material is not included in the article's Creative Commons license and your intended use is not permitted by statutory regulation or exceeds the permitted use, you will need to obtain permission directly from the copyright holder. To view a copy of this license, visit http://creativecommons.org/ licenses/by/4.0/

(C) The Author(s) 2020 